\title{
Clinical signs and outcomes of beef cattle undergoing cesarean section because of dystocia
}

\begin{abstract}
OBJECTIVE: To characterize signalment, clinical signs, reproductive history, surgical management, and outcomes of beef cattle undergoing cesarean section because of dystocia at a veterinary teaching hospital.
\end{abstract}

DESIGN: Retrospective case series with nested cohort study.

ANIMALS: 173 beef cattle admitted to a veterinary teaching hospital from 2001 through 2010 that underwent cesarean section because of dystocia.

PROCEDURES: Medical records were reviewed and information collected on cattle signalment; reproductive history; cause of dystocia; anesthetic protocol; surgical management; number, sex, and body weight of calves delivered (alive or dead); perioperative treatment; duration of hospitalization; and discharge status. A questionnaire regarding postoperative fertility was mailed to all owners, and owners who did not respond were contacted via telephone.

RESULTS: Overall mortality rate for calves was high, with $37.6 \%(62 / 165)$ of calves delivered dead or dying $\leq 24$ hours after cesarean section. Mortality rate was higher for female versus male calves and for calves from dams with signs of labor for $\geq 3$ hours versus $<3$ hours before hospital admission. Overall mortality rate for dams was low, with only 10 of $161(6.2 \%)$ dams failing to survive for $\geq 21$ days after hospital discharge. Postoperative fertility rate was acceptable, with $75 \%$ (44/59) of dams that were rebred after cesarean section giving birth to $\geq$ 1 live calf.

CONCLUSIONS AND CLINICAL RELEVANCE: Cesarean section was a clinically useful method for resolving dystocia in beef cattle, providing a high dam survival rate and an acceptable postoperative fertility rate. Beef cattle producers should seek veterinary assistance whenever clinical signs of dystocia are noticed, preferably within 6 hours after onset of parturition. 